\title{
Cloning and expression of BpMYC4 and BpbHLH9 genes and the role of BpbHLH9 in triterpenoid synthesis in birch
}

\author{
Jing Yin ${ }^{1,2}$, Xin Li ${ }^{1}$, Yaguang Zhan ${ }^{1,2^{*}}$, Ying Li ${ }^{1}$, Ziyue Qu ${ }^{1}$, Lu Sun ${ }^{1}$, Siyao Wang ${ }^{1}$, Jie Yang ${ }^{1}$ and Jialei Xiao ${ }^{3}$
}

\begin{abstract}
Background: Birch (Betula platyphylla Suk.) contains triterpenoids with anti-HIV and anti-tumor pharmacological activities. However, the natural abundance of these triterpenoids is low, and their chemical synthesis is costly. Transcription factors have the ability to regulate the metabolite pathways of triterpenoids via multi-gene control, thereby improving metabolite yield. Thus, transcription factors have the potential to facilitate the production of birch triterpenoids. Plant bHLH (basic helix-loop-helix) transcription factors play important roles in stress response and secondary metabolism.
\end{abstract}

Results: In this study, we cloned two genes, BPMYC4 and BpbHLH9, that encode bHLH transcription factors in Betula platyphylla Suk. The open reading frame (ORF) of BpMYC4 was 1452 bp and encoded 483 amino acids, while the ORF of BpbHLH9 was 1140 bp and encoded 379 amino acids. The proteins of BpMYC4 and BpbHLH9 were localized in the cell membrane and nucleus. The tissue-specific expression patterns revealed that BpMYC4 expression in leaves was similar to that in the stem and higher than in the roots. The expression of BpbHLH9 was higher in the leaves than in the root and stem. The expressions of BpMYC4 and BpbHLH9 increased after treatment with abscisic acid, methyl jasmonate, and gibberellin and decreased after treatment with ethephon. The promoters of BpMYC4 and BpbHLH9 were isolated using a genome walking approach, and 900-bp and 1064-bp promoter sequences were obtained for BpMYC4 and BpbHLH9, respectively. The ORF of BpbHLH9 was ligated into yeast expression plasmid pYES3 and introduced into INVSCl and INVScI1-pYES2-SS yeast strains. The squalene and total triterpenoid contents in the different INVScl1 transformants decreased in the following order INVScl1-pYES-SS-bHLH9 > INVSc11-pYES3-bHLH9 > INVSc11-pYES2- BpSS > INVScl-pYES2. In BpbHLH9 transgenic birch, the relative expression of the genes that encodes for enzymes critical for triterpenoid synthesis showed a different level of up-regulation compair with wild birch(control), and the contents of betulinic acid, oleanolic acid and betulin in bHLH9-8 transgenic birch were increased by $11.35 \%, 88.34 \%$ and $23.02 \%$ compared to in wild birch, respectively.

Conclusions: Our results showed that the modulation of BpbHLH9 by different hormones affected triterpenoid synthesis and triterpenoid contents. This is the first report of the cloning of BpbHLH9, and the findings are important for understanding the regulatory role of BpbHLH9 in the synthesis of birch triterpenoids.

Keywords: Betula platyphylla, BpMYC4, BpbHLH9, Expression characteristics, Triterpenoids

\footnotetext{
* Correspondence: yaguangzhan@126.com

${ }^{1}$ College of Life Science, Northeast Forestry University, Harbin 150040, China

${ }^{2}$ State Key Laboratory of Tree Genetic Breeding, Northeast Forestry University,

Harbin 150040, China

Full list of author information is available at the end of the article
} 


\section{Background}

Birch contains important secondary metabolites, including betulin, betulinic acid, and oleanolic acid. Betulinic and oleanolic acids show potential for use in anticancer and anti-HIV therapeutics [1]. However, the development of natural medicines is limited by the scarcity of natural drugs, and the effective use of natural plant resources is critical. Because of the low contents of plant secondary metabolites and their limited distribution in particular species, organs, tissues, and cells, improving the yields of plant secondary metabolites is important for the development of natural drugs [2].

Terpene biosynthesis is regulated by enzyme genes and transcription factors. Transcription factors regulate biosynthesis by changing the level of gene expression through alterations in transcription rate. Therefore, it is critical to study the transcription factors related to terpene biosynthesis [3]. The Basic helix-loop-helix (bHLH) transcription factors have been shown to be involved in plant growth and developmental processes (e.g., the formation of trichome, photomorphogenesis, and light signal transduction) along with stress response and secondary metabolism [4], and a few bHLH transcription factors have been identified that modulate the biosynthesis of plant terpenes [5].

MYC2 is a bHLH transcription factor known to play a primary role in the jasmonate (JA) signaling cascade [6]. The homologues of MYC2 played important roles in regulating the genes involved in sesquiterpene biosynthesis in Arabidopsis thaliana, Solanum lycopersicum, Catharanthus roseus, and Artemisia annua [7-10]. In Arabidopsis, MYC2 protein interacted with DELLA proteins to upregulate the expression of sesquiterpene synthase genes in flowers by integrating the gibberellin (GA) and JA signaling pathways [8]. In the presence of TTG1 (a WD40 repeat-containing protein) along with MYC2 protein formed a transcriptional regulatory complex with MYB protein, thereby activating the genes involved in anthocyanin biosynthesis [11]. In Catharanthus roseus, CrMYC2 was associated with the expression of several genes related to the biosynthesis of terpenoid indole alkaloid (TIA), and CrMYC2 bound with the cis-elements of the ORCA3 gene promoter to induce TIA accumulation [7]. Similarly, the NbMYC2a/b proteins from tobacco $(N$. tabacum) upregulated the ORCA-related NIC2 locus to enhance nicotine biosynthesis [12]. Recently, Bl (bitter leaf) and Bt (bitter fruit) were reported to regulate cucurbitacin triterpene accumulation in Cucumis sativus [13]. In Catharanthus roseus, BIS1 was reported to control the monoterpene (iridoid) branch of the monoterpene indole alkaloid pathway [14]. These transcription factors belong to the bHLH family. Furthermore, two bHLH transcription factors (NbbHLH1 and NbbHLH2) were shown to positively regulate the accumulation of nicotine through binding to the G-box elements of the putrescine $\mathrm{N}$ - methyltransferase promoter; in contrast, BbbHLH3 was found to be a negative regulator [15].

Although bHLH transcription factors have been characterized in Arabidopsis thaliana, Solanum lycopersicum (tomato), Catharanthus roseus, Artemisia annua, and other plants, none have been characterized in Betula platyphylla. Triterpenoids form from 2,3-oxidized squalene, a precursor of the mevalonate pathway. As important nodes in the metabolic pathways of triterpenoids, the key enzymes 3hydroxy-3-methyl glutaryl coenzyme A reductase (HMGR), farnesyl pyrophosphonate synthase (FPS), squalene synthase (SS), and squalene epoxidase (SE) were found to be directly involved in the synthesis of 2,3-oxidized squalene, and their contents and activities played important roles in regulating the synthesis and accumulation of terpenes $[16,17]$. We previously showed that the promoters of HMGR, FPS, SS, and SE in B. platyphylla contained G-box elements regulated by bHLH transcription factors, and these bHLH transcription factors may influence the synthesis and accumulation of triterpenoids [2]. Our past work also suggested that $S S$ and $S E$ in B. platyphylla transformed the INVScl1 strain of yeast, as indicated by the significant increase in squalene content caused by the INVScl1 transformants [2]. Based on these previous results, we selected two transcription factors, MYC4 and bHLH9, from our laboratory transcriptome database. These transcription factors were demonstrated to respond to methyl jasmonate (MeJA) signaling and may play a role in the synthesis of birch triterpenes. We then cloned the full lengths of the genes encoding MYC4 and bHLH9 (BpMYC4 and BpbHLH9, respectively) and analyzed their tissue-specific expression patterns after treatment with different phytohormones. In addition, we transferred BpbHLH9 to the INVScl1-pYES2BpSS yeast strain in an attempt to gain a greater understanding of the regulatory roles of BpMYC4 and BpbHLH9 in birch triterpenoid biosynthesis.

\section{Results \\ Cloning and analysis of BpMYC4 and BpbHLH9}

Special primers were designed based on the nucleotide sequence in the transcriptome database of B. platyphylla (Additional file 1: Table S1). The full lengths of BpMYC4 and BpbHLH9 from B. platyphylla were isolated by realtime RT-PCR. The BpMYC4 cDNA sequence length was $1564 \mathrm{bp}$ and contained a 1452-bp open reading frame (ORF) encoding 483 amino acids (Additional file 2: Figure S1). Similarly, a BpbHLH9 cDNA sequence of 1243 bp was obtained with a 1140-bp ORF encoding a protein of 379 amino acids (Additional file 3: Figure S2).

The conserved domain of the BpMYC4-encoded protein was predicted using the Conserved Domains function in BLAST. The results indicated that the protein contained a bHLH-MYC-N-specific hit within a.a. 25-210, corresponding to the N-terminal region of a family of MYB and 
MYC transcription factors. In several plant species, members of this family regulate phenylpropanoid biosynthesis. The HLH domains, which bind DNA, were located further downstream at a.a. 312-362, 316-364, and 313359 (Fig.1). The physicochemical properties of the BpMYC4 amino acid sequences were analyzed using the Protparam bioinformatics tool of the ExPASy website; the results indicated a molecular mass of $54.41 \mathrm{kDA}$ and an isoelectric point of 5.78. The BpMYC4-deduced protein was found to be unstable and hydrophilic. The amino acid sequence of BpMYC4 was approximately $70 \%$ homologous with the bHLHs of Theobroma cacao, Populus trichocarpa, and Populus euphratica. The multiple alignment of the BpMYC4-encoded amino acid sequence with other bHLH proteins is shown in Fig.1 (a, b). The neighbor-joining phylogenetic tree, which was constructed using MEGA 6.0 software (Fig. 2), indicates a strong correlation between the BpMYC4-encoded amino acids and the amino acids of Citrus sinensi (KDO86574.1). The secondary structure of the BpMYC4 protein revealed using GOR4 software suggested that BpMYC4 consists of $\alpha$-helices (37.74\%), extended strands (17.39\%), and random coils (45.13\%).

The conserved domain of the BpbHLH9-deduced protein was predicted using the Conserved Domains function in BLAST. The results indicate that the protein contains a HLH domain at a.a. 180-231, 178-235, and 186-236, with
E-box and N-box elements in the vicinity of the HLH domain. The HLH domain is found in specific DNAbinding proteins that act as transcription factors. The physicochemical properties of the BpbHLH9 amino acid sequences were analyzed using the Protparam bioinformatics tool of the ExPASy website, revealing a molecular mass of $42.90 \mathrm{kDA}$ and an isoelectric point of 6.10. The BpbHLH9-encoded protein was found to be unstable and hydrophilic. The amino acid sequence of BpbHLH9 was approximately $60 \%$ homologous with the bHLHs of Theobroma cacao, Glycine soja, and Phaseolus vulgaris. The multiple alignment of the amino acid sequence encoded by BpbHLH9 with other bHLH proteins is shown in Fig. 3. The neighbor-joining phylogenetic tree constructed using MEGA 6.0 software (Fig. 4) indicated good correlation between the BpbHLH9-encoded amino acids with bHLH57 from Pyrus x bretschneideri and Malus domestica.The secondary structure of the BpbHLH9 protein determined using the GOR4 program indicated that BpbHLH9 consists of $\alpha$-helixes (42.74\%), extended strands (12.40\%), and random coils (44.85\%).

\section{Isolation and analysis of BpMYC4 and BpbHLH9 promoter sequences}

We obtained BpMYC4 and BpbHLH9 gene promoters from $B$. platyphylla using a genome walking approach.

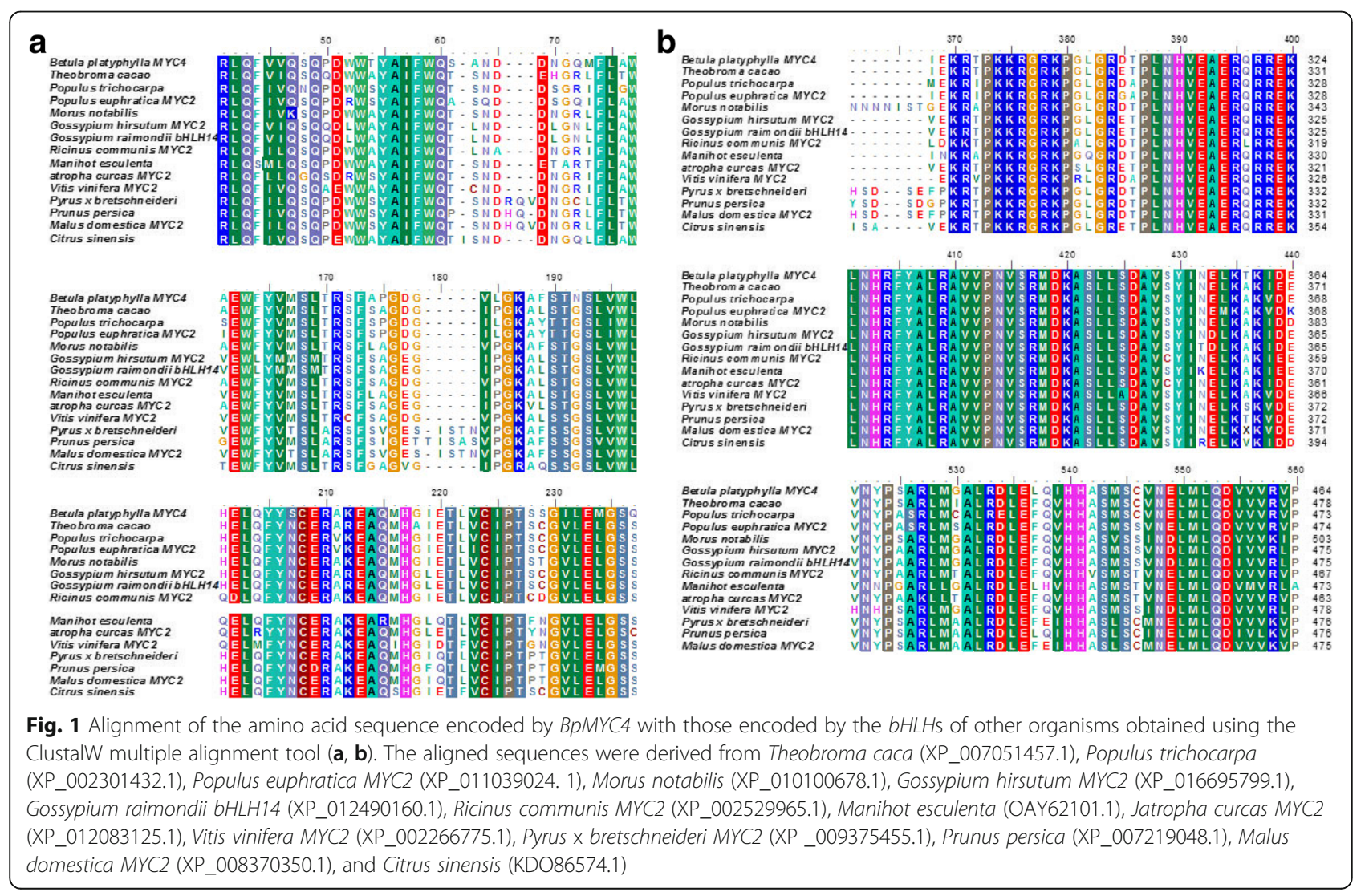




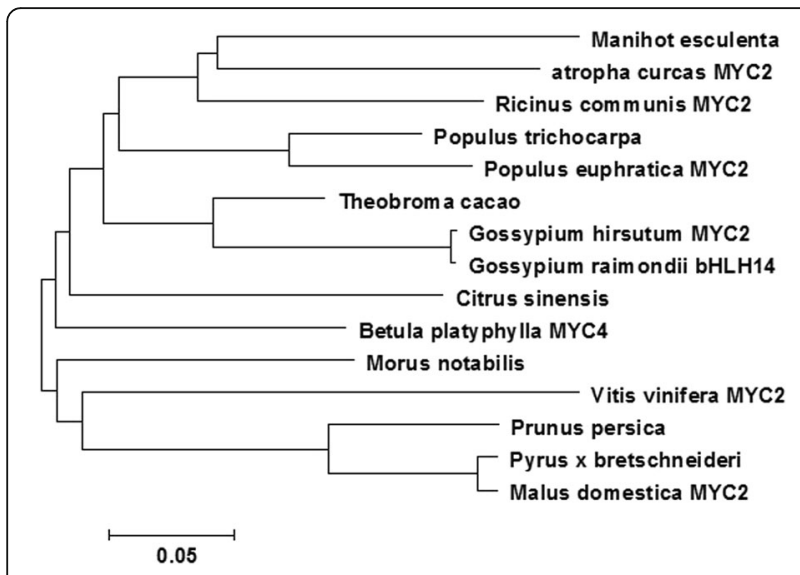

Fig. 2 Neighbor-joining phylogenetic tree of BpMYC4 from Betula platyphyll and other bHLHs constructed using MEGA 6.0 software. The scale bar represents 0.05 amino acid substitutions per site
The length of the BpMYC4 gene promoter was $900 \mathrm{bp}$, and the $5^{\prime}$-untranslated region (5'UTR) was located at the +458-bp site of the promoter. Similarly, the -444-bp site of the $\mathrm{BpbHLH} 9$ gene promoter sequence (length $=1064 \mathrm{bp}$ ) also contained a 5'UTR sequence (Additional file 4: Figures S3 and S4). The two promoter sequences were analyzed using PlantCARE software (Tables 1 and 2). The results indicated that the promoter regions of BpMYC4 and BpbHLH9 contained multiple eukaryotic cis-acting elements, including TATA and CAAT boxes. In the BpMYC4 promoter sequence, one abscisic acid response element (ABRE) was found at the +773-bp site, three HSE response elements were located at $-487,-677$, and $-676 \mathrm{bp}$, one drought response element (MBS) was located at $-599 \mathrm{bp}$, two TC-rich repeating elements involved in defense and stress response were located at +156 and $-886 \mathrm{bp}$, one endosperm expression element (GCN4)

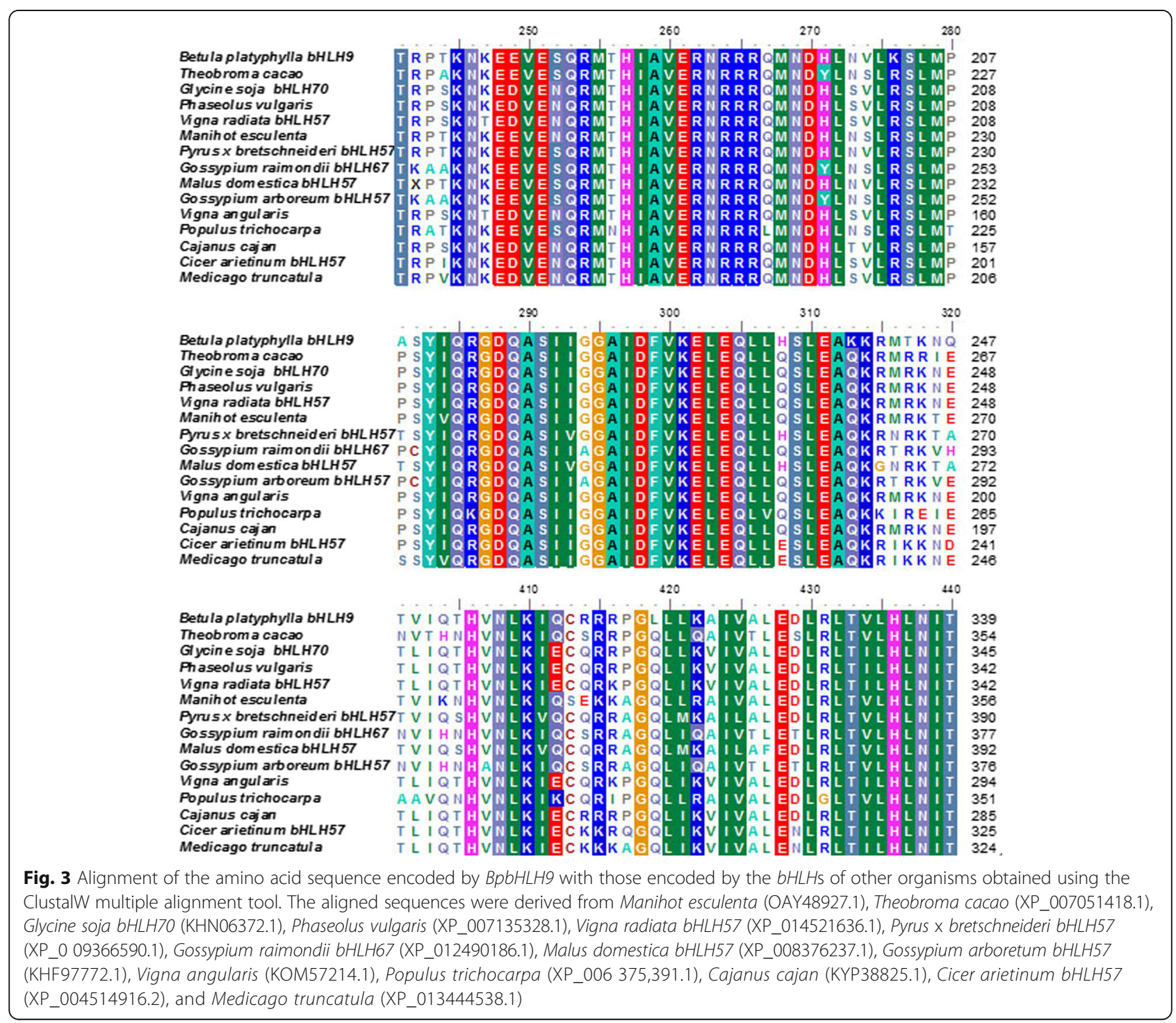




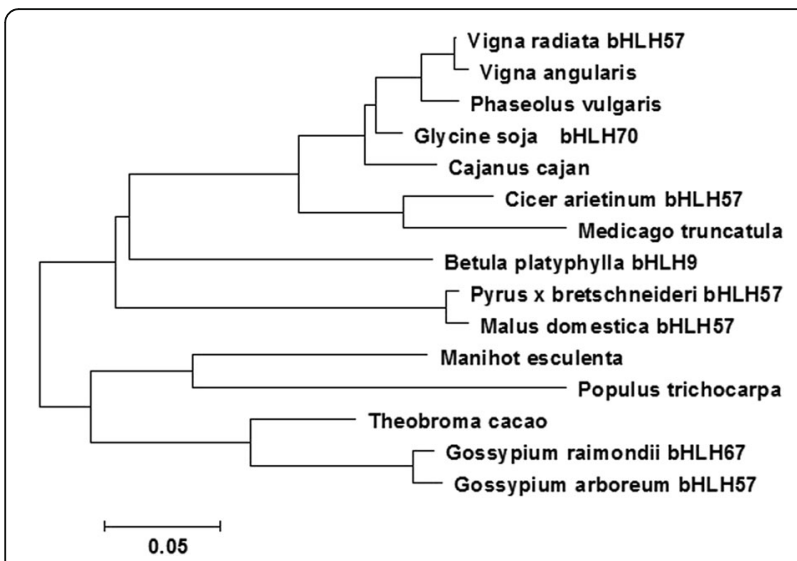

Fig. 4 Neighbor-joining phylogenetic tree of BpbHLH9 from Betula platyphylla and other bHLHs constructed using the MEGA 6.0 software program. The scale bar represents 0.05 amino acid substitutions per site

was found at $-321 \mathrm{bp}$, and one cis-acting element associated with circadian rhythm was located at $-432 \mathrm{bp}$.

BpbHLH9 also contained plant hormone-response elements, heat-response elements, drought-response elements, defense- and stress-response elements, and endospermexpression elements, including ABRE (involved in abscisic acid response), the GARE motif (involved in GA response), and TCA [involved in salicylic acid (SA) response], which were located at $+146,-791,-785$ and +128 , respectively. In addition, the BpbHLH9 promoter contained multiple anaerobic and cryogenic regulatory elements. The BpMYC4 and BpbHLH9 promoters were predicted to contain MBS elements that play an important role in regulating the synthesis of secondary metabolites. The presence of these regulatory elements indicated that the cloned sequence conformed to the basic characteristics of the eukaryotic gene promoter, and that the expressions of the BpMYC4 and BpbHLH9 genes of B. platyphylla were regulated by these elements and responded to a variety of plant hormones and adversities stress.

\section{Expression patterns of BpMYC4 and BpbHLH9 under phytohormone treatments}

RT-PCR experiments were conducted to study the BpMYC4 and BpbHLH9 expression patterns in different tissues of $B$. platyphylla. The results showed that the expression of $B p M Y C 4$ in leaves was similar to the expression in the stem and higher than the expression in roots (Fig. 5a). The expression of BpbHLH9 was higher than in the root and stem tissues (Fig. 5b). Different expression patterns were obtained by treating BpMYC4 and BpbHLH9 with different hormones (Fig. 6). The expression of BpMYC4 was up-regulated by treatment with abscisic acid (ABA; $10 \mu \mathrm{M})$, MeJA (100 M), GA $(100 \mathrm{mg} / \mathrm{L})$, or $\mathrm{SA}(50 \mathrm{mg} / \mathrm{L})$ for $12 \mathrm{~h}$. The greatest

Table 1 Putative cis-acting regulatory elements identified in the promoter sequence of BpMYC4 using the PlantCARE database

\begin{tabular}{|c|c|c|c|}
\hline Cis element & Position & Sequence & Function of site \\
\hline 5'UTR Py-rich stretch & +458 & TाTСТTСТCT & cis-acting element conferring high transcription levels \\
\hline ABRE & +773 & CGCACGTGTC & cis-acting element involved in abscisic acid response \\
\hline ATGCAAAT motif & -540 & ATACAAAT & $\begin{array}{l}\text { cis-acting regulatory element associated with the } \\
\text { TGAGTCA motif }\end{array}$ \\
\hline CAAT-box & $\begin{array}{l}-65,-54,+265,+829 \\
+137,+669,-409 \\
-831,-66,-555,+287 \\
-830,+219,+828,+538\end{array}$ & $\begin{array}{l}\text { CAATT, } \\
\text { CAAAT, } \\
\text { CAAT, } \\
\text { CCAAT }\end{array}$ & $\begin{array}{l}\text { common cis-acting element in promoter and enhancer } \\
\text { regions }\end{array}$ \\
\hline G-Box & $\begin{array}{l}-376,-775,-376,+843 \\
-775\end{array}$ & $\begin{array}{l}\text { CACGT, } \\
\text { CACGTC }\end{array}$ & cis-acting regulatory element involved in light response \\
\hline GCN4_motif & -321 & TGTGTCA & cis-regulatory element involved in endosperm expression \\
\hline HSE & $-478,-677,-676$ & $\begin{array}{l}\text { AGAAAATTCG, } \\
\text { AAAAAATTTC }\end{array}$ & cis-acting element involved in heat stress response \\
\hline MBS & -599 & TAACTG & MYB binding site involved in drought-inducibility \\
\hline Sp1 & $+49,-642,-629,-661$ & $C C(G / A) C C C$, GGGCGG & light-responsive element \\
\hline TATA-box & $\begin{array}{l}-35,+37,+39,+40,+41,+43, \\
+157,-210,-211,-212,-213 \\
+214,-223,+238,-241,+251 \\
-252,+253,+278,+296,-299 \\
-346,+348,+349,+350,+351 \\
-503,-532,-541,-543,-545 \\
+655,+762,+793,+798,-801\end{array}$ & $\begin{array}{l}\text { TATATAAATC, TCTATATATT, } \\
\text { TATATATA, ATATAT, TATA, } \\
\text { TITA, TATAAAAT, TATAAAA, } \\
\text { TATAAA,TATAA, TATACA }\end{array}$ & $\begin{array}{l}\text { core promoter element located next to }-30 \text { of transcription } \\
\text { start }\end{array}$ \\
\hline TC-rich repeats & $+156,-886$ & ATTTCTTCA, GTTTCTTAC & cis-acting element involved in defense and stress response \\
\hline TGG-motif & -619 & GGTTGCCA & part of a light-responsive element \\
\hline circadian & -432 & CAANNNNATC & cis-acting regulatory element involved in circadian control \\
\hline
\end{tabular}


Table 2 Putative cis-acting regulatory elements identified in the promoter of BpbHLH9 by PlantCARE databases

\begin{tabular}{|c|c|c|c|}
\hline Cis element & Position & Sequence & Function of site \\
\hline $4 \mathrm{cl}-\mathrm{CMA} 2 \mathrm{~b}$ & +699 & TCTCACCAACC & light-responsive element \\
\hline 5'UTR Py-rich stretch & $\begin{array}{l}444,-575,-577,-579,-581 \\
+877,+881,+883,+885,+887, \\
+889,+891,+893,+895,+897, \\
+899,+901,+903,+905\end{array}$ & 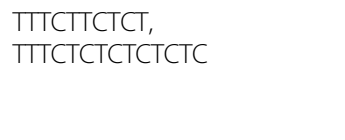 & cis-acting element conferring high transcription levels \\
\hline ABRE & +146 & CACGTG & cis-acting element involved in abscisic acid response \\
\hline ACE & +147 & ACGTGGA & cis-acting element involved in light response \\
\hline ARE & $-317,-665$ & TGGTTT & cis-acting regulatory element essential for anaerobic induction \\
\hline ATGCAAAT motif & -352 & ATACAAAT & cis-acting regulatory element associated with the TGAGTCA motif \\
\hline Box 1 & +231 & TTTCAAA & light-responsive element \\
\hline CAAT-box & $\begin{array}{l}+122,+316,-267,-1039,-188, \\
+644,+331,-1044,-187,+643 \\
+275,+1043,+206,-750,-352 \\
-1045\end{array}$ & $\begin{array}{l}\text { CAAT, } \\
\text { CAATT, } \\
\text { CAAAT, }\end{array}$ & common cis-acting element in promoter and enhancer regions \\
\hline CCAAT-box & -842 & CAACGG & MYBHv1 binding site \\
\hline G-Box & $+146,-389$ & CACGTG, CACATGG & cis-acting regulatory element involved in light response \\
\hline GAG-motif & $-58,-879,-707$ & AGAGAGT & part of a light-responsive element \\
\hline GARE-motif & -791 & AAACAGA & gibberellin-responsive element \\
\hline GATA-motif & +19 & GATAGGG & part of a light-responsive element \\
\hline GCN4_motif & $-141,-516,-490$ & TGTGTCA & element involved in endosperm expression \\
\hline HSE & +262 & AGAAAATTCG & cis-acting element involved in heat stress response \\
\hline I-box & +19 & GATAGGG & part of a light-responsive element \\
\hline L-box & +699 & TCTCACCAACC & part of a light-responsive element \\
\hline LTR & +102 & CCGAAA & cis-acting element involved in low-temperature response \\
\hline MBS & +605 & CAACTG & MYB binding site involved in drought-inducibility \\
\hline Skn-1_motif & $-140,-489,+191$ & GTCAT & cis-acting regulatory element required for endosperm expression \\
\hline Sp1 & +763 & $\mathrm{CC}(\mathrm{G} / \mathrm{A}) \mathrm{CCC}$ & light-responsive element \\
\hline GCN4_motif & $-141,-516,-490$ & TGTGTCA & element involved in endosperm expression \\
\hline TATA-box & $\begin{array}{l}-80,-181,+239,+344 \\
-439,-571,-724,-732,-733, \\
-734,-735,-830 \\
+852,+944,-946,-947,-956, \\
-964,-990,-1058\end{array}$ & $\begin{array}{l}\text { CCTATAAATT, TाTA, } \\
\text { TATA, ATATAT,TAATA }\end{array}$ & core promoter element around of transcription start \\
\hline TC-rich repeats & $+397,+814$ & ATTITCTTCA & cis-acting element involved in defense and stress response \\
\hline TCA-element & $+128,-785$ & CCATCTIITI, CAGAAAAGGA & cis-acting element involved in salicylic acid response \\
\hline TCT-motif & +775 & TCTTAC & part of a light-responsive element \\
\hline
\end{tabular}

change in expression was observed in response to MeJA treatment (Fig. 6a), which resulted in a 76-fold increase in BpMYC4 transcripts during the 12-h treatment. BpMYC4 transcripts were also up-regulated 2.3 and 3.6 times compared to the control after 24 and $48 \mathrm{~h}$ of induction with MeJA, respectively. In contrast, the expression of BpMYC4 was down-regulated by treatment with the other three hormones for 24 and $48 \mathrm{~h}$. After $12 \mathrm{~h}$ of treatment with MeJA, GA, SA and ABA, BpMYC4 was up-regulated by 76, 6.7, 5.9 and 5.4 times compared to the control group, respectively (Fig. 6a-d). The expression of $\mathrm{BpbHLH} 9$ was increased 5.2-fold by MeJA treatment for $12 \mathrm{~h}$ compared to the control (Fig. 6b). However, the expression patterns obtained after treatment with the other hormones were different from those observed for BpMYC4. For BpbHLH9, treatment with ABA and GA for $12 \mathrm{~h}$ resulted in 2.6- and 1.9-fold decreases in expression compared to the control, respectively. Additionally, the expressions of BpMYC4 and BpbHLH9 transcripts were decreased $4 \%$ and $1 \%$ upon ethephon treatment for 6 and $24 \mathrm{~h}$ respectively (Fig. 6e), compared to the control. Interestingly, treatment with SA caused a drastic decrease in BpbHLH9 expression, which reached the minimum after $24 \mathrm{~h}$; compared to the control group, the expression of $\mathrm{BpbHLH} 9$ was reduced by $99 \%$ after $24 \mathrm{~h}$ of SA treatment (Fig. 6c). These results suggested that SA negatively 

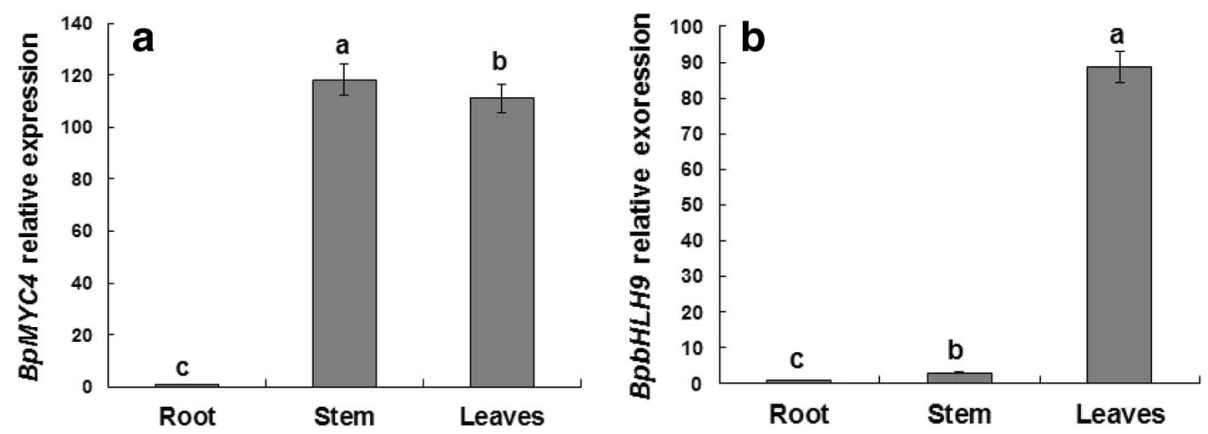

Fig. 5 Tissue-specific expressions of BpMYC4 (a) and BpbHLH9 (b) in birch seedlings. The relative expressions of BpMYC4 and BpbHLH9 were quantified by quantitative RT-PCR. Reported values are means of three replicates, and the error bars were obtained from multiple replicates. The letters in Fig. 5 indicate a significant difference at the 0.05 level

regulated the expression of BpbHLH9. Furthermore, the expression patterns indicated that the two studied bHLH transcription factors had different functions and regulation mechanisms related to plant growth and metabolism.

\section{BpMYC4 and BpbHLH9 localization in the nucleus and cell} membrane

We investigated the subcellular localization of BpMYC4 and BpbHLH9 in living cells through transient expression assays using onion epidermal cells with constructs that only express GFP, the BpMYC4/GFP fusion, and the BpbHLH9/GFP fusion. Fluorescence microscopy showed that the BpMYC4/GFP and BpbHLH9/GFP fusion proteins were targeted in the nucleus and cell membrane. In contrast, the control (GFP only) distributed throughout the cell (Fig. 7). These findings indicated that BpMYC4 and BpbHLH9 localized in the nucleus and cell membrane.

\section{Analysis of squalene and total triterpenoid products accumulated in INVScl1 transformants}

To study the function of the BpbHLH9 gene, recombinant yeast and control yeast were induced by galactose for $12 \mathrm{~h}$, and the squalene synthase product was extracted and analyzed by high-performance liquid chromatography (HPLC).

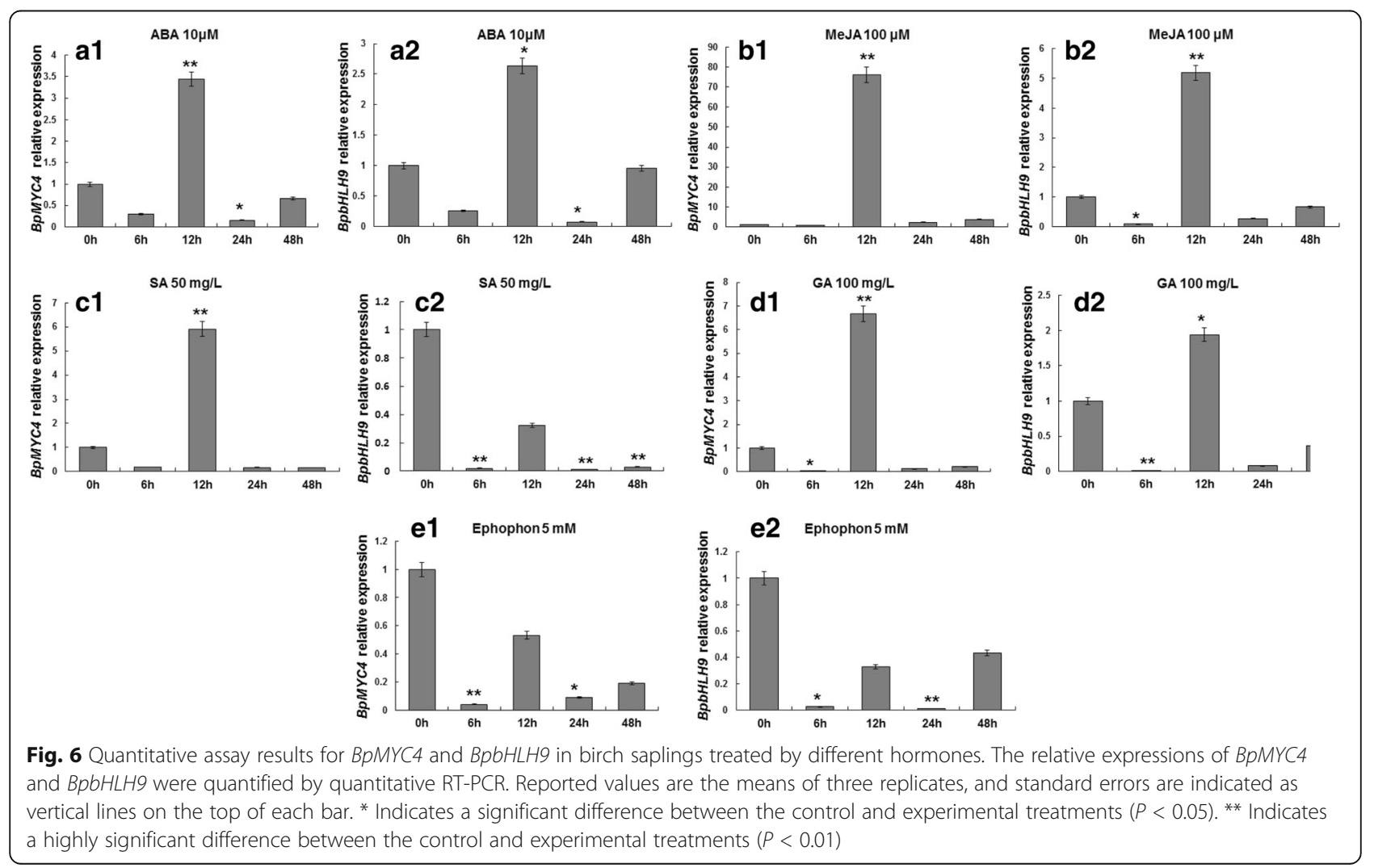




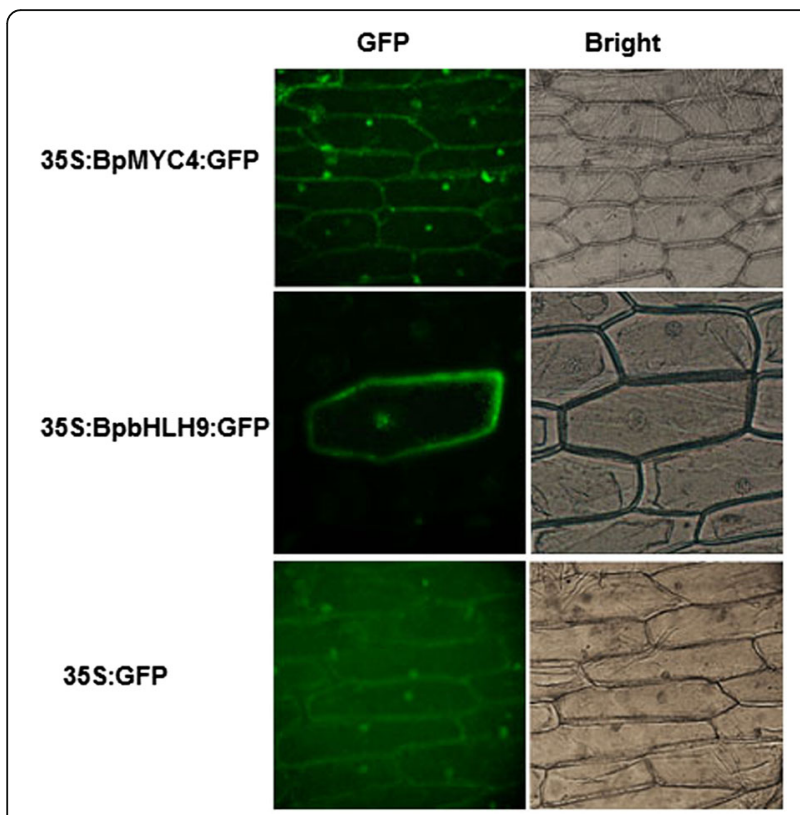

Fig. 7 Subcellular localization of BpMYC4/GFP and BpbHLH9/GFP fusion proteins in onion epidermal cells

We plotted the standard curve of squalene and determined the regression equation to be $y=10,000,000 \times-12,009$ $\left(R^{2}=0.9994\right)$. After culturing for $12 \mathrm{~h}$, the HPLCdetermined squalene content of pYES-BpSS-BpbHLH9 expression yeast cells $(0.328 \mathrm{mg} / \mathrm{g})$ was approximately 2.45 times that in pYES2-BpSS expression yeast cells $(0.134 \mathrm{mg} / \mathrm{g}), 1.98$ times that in pYES3-BpbHLH9 expression yeast cells $(0.165 \mathrm{mg} / \mathrm{g})$, and 3.99 times that in the empty vector control yeast cells $(0.082 \mathrm{mg} / \mathrm{g}$, Fig. 8). These results suggested that BpbHLH9 was involved in triterpenoid synthesis.

To further study the function of BpbHLH9, the recombinant yeast and control yeasts were inoculated for $12 \mathrm{~h}$ followed by the extraction of triterpenoids and determination of total triterpenoid content. The standard curve of oleanolic acid was drawn, and the regression equation was determined as $y=43.044 \times-0.6438\left(R^{2}=\right.$ 0.997). The results indicate that when BpbHLH9 or BpSS was transferred into INVScl1 yeast, the content of total triterpenoids in the INVScl1 transformants was significantly increased. When BpbHLH9 and BpSS genes were co-transformed into yeast cells, the total triterpenoid content was further enhanced. The total triterpenoid content in INVScl-pYES-SS-bHLH9 yeast cells was $54.23 \mathrm{mg} / \mathrm{g}$, $10.39 \%$ higher than that in the control yeast (INVSclpYES2). The total triterpenoid content in INVScl-pYES2SS and INVScl-pYES3-bHLH9 yeasts were increased by $4.49 \%$ and $6.00 \%$, respectively, compared to in the control yeast (Fig. 9). These results indicated that BpbHLH9 gene was involved in the syntheses of squalene and triterpenoids.

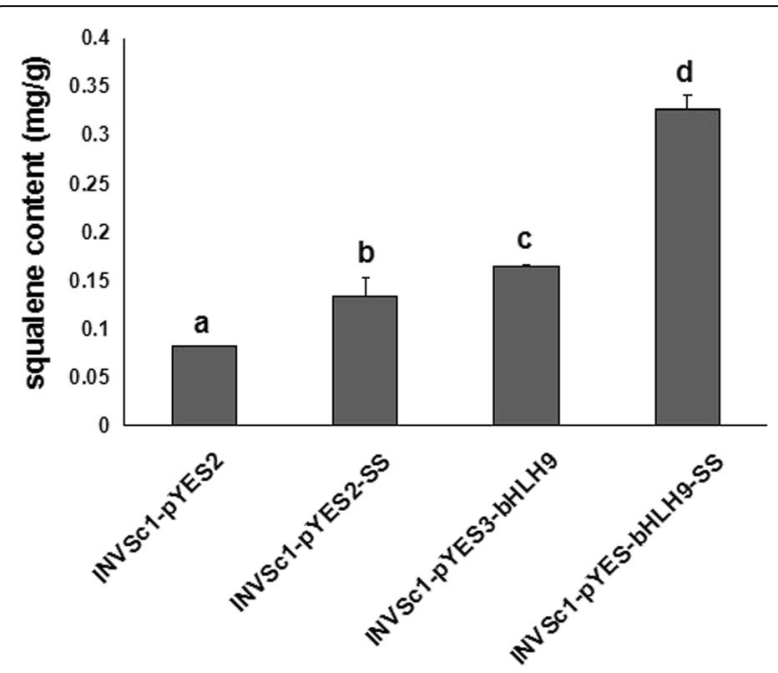

Fig. 8 Squalene contents in the cells of the control and transgenic yeasts. The reported values are means of three replicates, and the vertical line on top of each bar indicates standard error. The letters in Fig. 8 indicate a significant difference at the 0.05 level

\section{Relative expression of genes related to triterpene} synthesis in transgenic birch seedlings

To study the role of BpbHLH9 in triterpenoid synthesis in birch, we obtained BpbHLH9 transgenic seedlings of Birch. (Additional file 5: Figures S5, S6 and S7). The expressions of genes related to triterpene synthesis (HMGR, FPS, SS, SE, BPY and BPW) in the transgenic plants (lines 7 and 8) with high over-expression of BpbHLH9 were detected by real time RT-PCR. The results are shown in Fig. 10. The expressions of HMGR, FPS, SS, SE, BPY and BPW showed different degrees of up-regulation in the bHLH9-7 and bHLH9-8 transgenic plants lines. The greatest change was observed in the expression of $B P Y$, which was 26.15 times

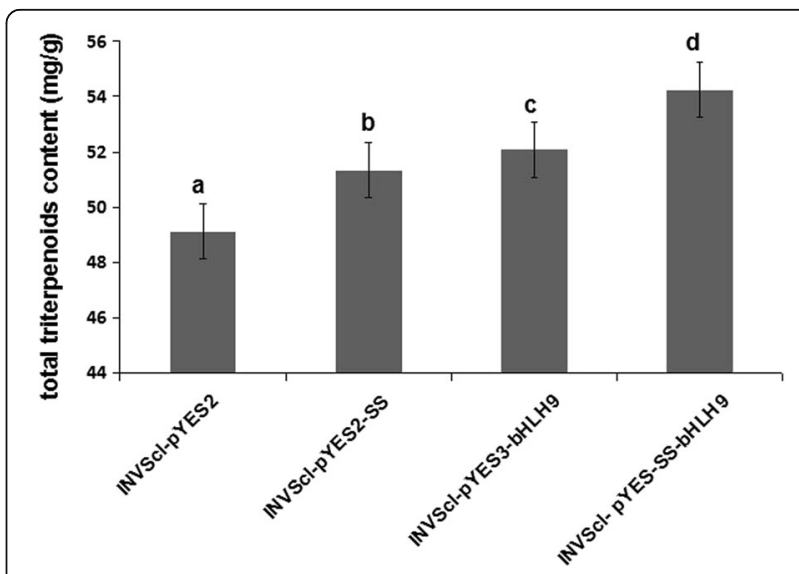

Fig. 9 Total triterpenoid contents in the cells of the control and transgenic yeasts. The reported values are the means of three replicates, and the vertical line on top of each bar indicates standard error. The letters in Fig. 9 indicate a significant difference at the 0.05 level 


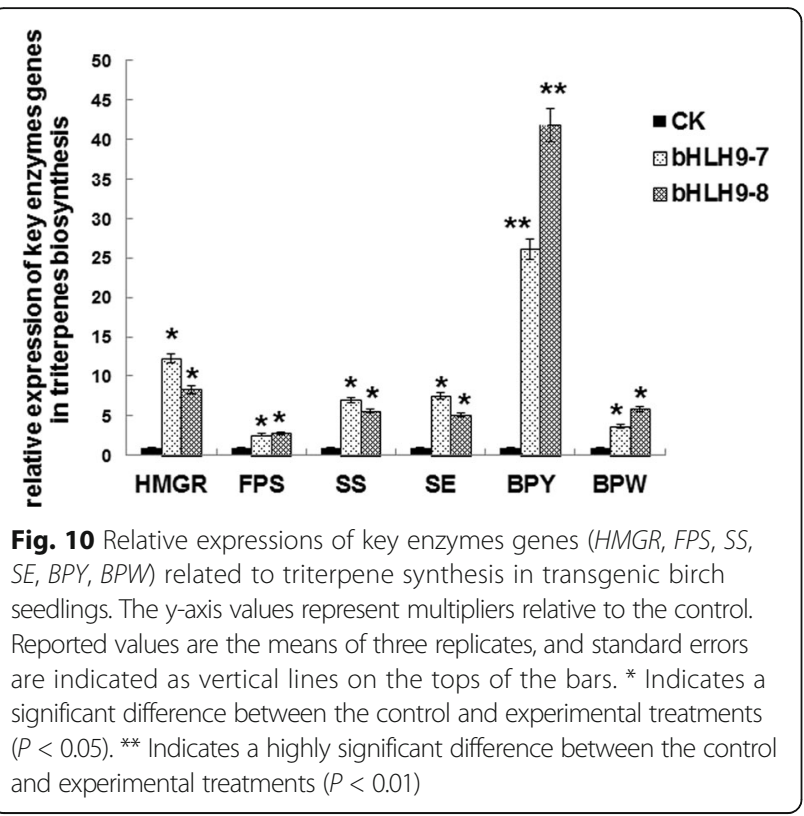

and 41.76 times that of the wild birch (control), respectively, followed by the expressions of $H M G R, S S, S E, B P W$, and FPS.

\section{HPLC analysis of betulinic acid, oleanolic acid and betulin} in $\mathrm{BpbHLH9}$ transgenic birch

To further determine the function of BpbHLH9 gene in triterpene synthesis, the callus was induced by stem segments of bHLH9-7 and bHLH9-8 lines that greatly overexpress BpbHLH9. The triterpenoids were then extracted, and the contents of betulinic acid, oleanolic acid, and betulin in the callus were determined. The standard curves of betulinic acid, oleanolic acid and betulin were drawn, and the respective regression equations were determined as follows: $y_{1}=9 \times 10^{6} x+68,020\left(R^{2}=\right.$ $0.9994) ; \quad y_{2}=10^{7} x+43,301\left(R^{2}=0.9993\right) ;$ and $y_{3}=10^{7} x+63,763\left(R^{2}=0.9995\right)$. The results showed that the contents of betulinic acid, oleanolic acid and betulin in the callus were higher in the bHLH9-7 and bHLH9-8 lines than in the wild type. Compared to the wild type birch (the control), the contents of betulinic acid, oleanolic acid and betulin in bHLH9-7 transgenic callus were increased by $9.49 \%, 45.35 \%$ and $9.56 \%$, respectively. The contents of betulinic acid, oleanolic acid and betulin in bHLH9-8 transgenic birch callus were increased by $11.35 \%, 88.34 \%$ and $23.02 \%$ compared to in wild birch, respectively. Among the three tested compounds, the overexpression of BpbHLH9 increased the content of oleanolic acid the most, followed by betulin and betulinic acid (Fig. 11). These results showed that BpbHLH9 gene was involved in the synthesis of triterpenoids.

\section{Discussion}

Several transcription factors in the bHLH family have been reported to regulate the biosynthesis of different secondary metabolites in various plant species [7-10, 13]. These identified TFs controlled the metabolic flow through the biosynthetic pathway by positively or negatively regulating the expressions of genes that encode key enzymes [18]. TFs in the bHLH family have been shown to play important roles in the biosynthesis of triterpene in plants [5]; for example, TSAR1 and TSAR2 modulated the biosynthesis of triterpene saponin in Medicago truncatula [4], and BIS2 was critical for the generation of monoterpenoid indole alkaloid in Catharanthus roseus [19]. In this study, we first cloned the full lengths of genes encoding transcription factors MYC4 and bHLH9 in B. platyphylla using RT-PCR; these genes were temporarily named BpMYC4 and BpbHLH9, respectively. NCBI BLAST alignment results showed that the amino acid sequence encoded by BpMYC4 is approximately $70 \%$ homologous with the bHLHs of Theobroma cacao, Populus trichocarpa, and Populus euphratica. The conserved domains indicated that the polypeptide sequence encoded by BpMYC4 contained a bHLH-MYC-N-specific hit. The biosyntheses of phenylpropanoids in tobacco [20], Arabidopsis and Petunia [21] were shown to be regulated by members of the MYB and MYC families. We hypothesize that $B p M Y C 4$ is involved in secondary metabolism of phenylpropanoids. The amino acid sequence encoded by BpbHLH9 was approximately $60 \%$ homologous with the bHLHs of Theobroma cacao, Glycine soja, and Phaseolus vulgaris. The polypeptide sequence encoded by BpbHLH9 contained a HLH domain, which is characteristic of DNA-binding proteins that serve as transcription factors. Thus, we think that BpMYC4 and BpbHLH9 are two new genes.

We obtained the promoters of BpMYC4 and BpbHLH9 from $B$. platyphylla using a genome walking approach; the corresponding fragment lengths were 900 and 1064 bp, respectively. The analyses of the promoter sequences are detailed in Additional file 4: Figures S3 and S4). The BpMYC4 and BpbHLH9 promoters contained many RNA polymerase binding sites essential for gene transcription (e.g., TATA and CAAT boxes) along with stress-related cis-elements that allow the BpMYC4- and BpbHLH9encoded transcription factors to adapt to the environment. The promoters also contained some light-controlling elements and plant hormone-response elements (e.g., ABRE and the GARE motif), indicating that BpMYC4 and $B p b H L H 9$ were regulated by plant hormones. Hiroshi Abe et al. [22] confirmed that AtMYC2, a transcription factor in the bHLH family, was found to act as a transcriptional activator in ABA signaling in Arabidopsis. Analyzing the expression patterns of BpMYC4 and BpbHLH9 revealed that their expressions were significantly increased after $12 \mathrm{~h}$ of treatment with GA $(100 \mathrm{mg} / \mathrm{L})$ or ABA $(10 \mu \mathrm{M})$. 

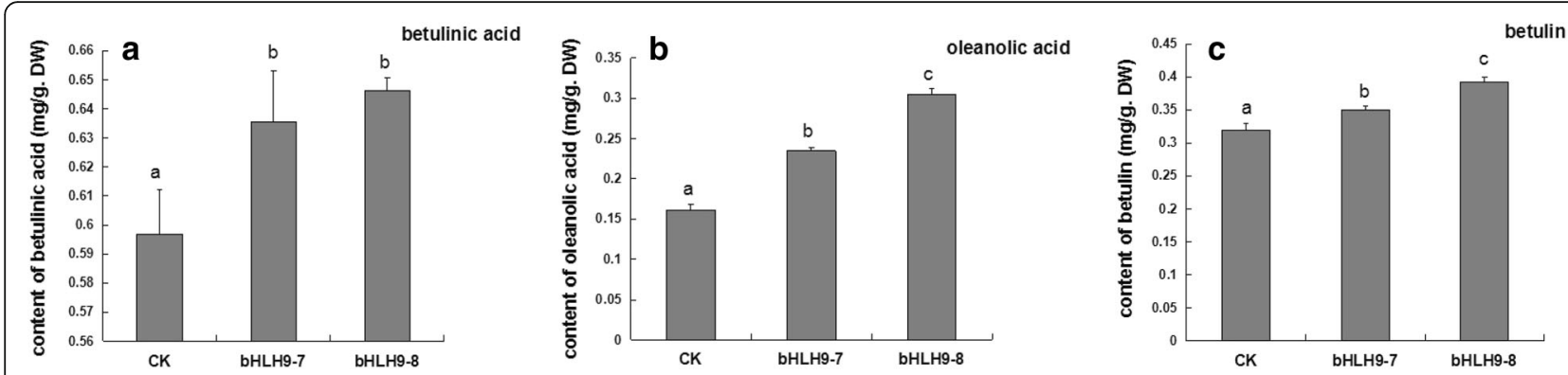

Fig. 11 The contents of betulinic acid, oleanolic acid and betulin in BpbHLH9 transgenic birch. The reported values are the means of three replicates, and the vertical line on top of each bar indicates standard error

In plants, MeJA and SA were shown to act as signaling molecules of biotic and abiotic stresses [23, 24]; these hormones had either inhibitory or promoting effects that manifested in the form of morphological or physiological changes or defense response [24, 25]. In defense response, plants produce a large quantity of secondary metabolites, including terpenoids. Thus, the biosynthesis of secondary metabolites can be modulated through treatment with these signaling molecules. In a previous study, we demonstrated that MeJA treatment significantly enhanced the contents of total triterpenes, betulinic acid and oleanolic acid in birch and up-regulated the expressions of FPS, SS, $S E, B P Y$ and $B P W$, which are involved in triterpene biosynthesis $[1,2,16,17]$. In this study, after $12 \mathrm{~h}$ of MeJA treatment, the expressions of both BpMYC4 and BpbHLH9 in $B$. platyphylla were significantly up-regulated. These results were similar to the expression patterns of BpSS and BpSE observed in our previous study [2], indicating that BpMYC4 and BpbHLH9 acted as signaling molecules in the biosynthesis of triterpenes in $B$. platyphylla. We hypothesize that MeJA induced BpMYC4 and BpbHLH9 accumulation and regulated the downstream expressions of $B p S S$ and $B p S E$ by binding G-box cis-elements. Our previous study demonstrated that BpSS and BpSE contained G-box of bHLH regulatory element [2]. Chatel et al. [26] reported that MeJA induced the accumulation of the mRNA that encodes the transcription factor CrMYC1, which is in the bHLH family and specifically binds the G-box element in yeast [27]. Li et al. demonstrated that MYC2 inhibited the activation of genes related to terpene synthase [28]. Clearly, bHLH factors were synthesized de novo in response to MeJA, thereby conferring the MeJA-responsive expression of secondary response genes via binding to Gbox-like sequences in their promoters [27]. However, in this study, the expression of BpbHLH9 decreased after SA treatment. A previous study demonstrated that suppression of the JA pathway by SA functions downstream of the E3 ubiquitin-ligase Skip-Cullin-F-box complex $\mathrm{SCF}^{\mathrm{COI1}}$, which targeted JASMONATE ZIM-domain transcriptional repressor proteins (JAZs) for proteasome- mediated degradation [29]. Collectively, the above data suggested that JA signaling was inhibited by the SA pathway as a result of the negative regulation of BpbHLH9 transcriptional factor expression. Ethylene has been reported to enhance anthocyanin, flavonoid, and stilbenoid production in grape (Vitis vinifera) cell cultures by upregulating the corresponding biosynthetic genes [30]. Interestingly, in this study, the mRNA expression of BpMYC4 and BpbHLH9 decreased after treatment with ethephon, suggesting that BpMYC4 and BpbHLH9 acted as negative regulators with specificity for the ethephon signaling pathway. This process might be responsible for the biosynthesis of secondary metabolites.

TF (transcription factors) have been shown to initiate the expressions of terpenoid synthesis-related genes by binding to the cis-acting elements of the promoters of downstream genes. The transcription factors for terpene biosynthesis specifically bound to the G-Box region to initiate the production of genes related to triterpenoid synthesis [31]. Moreover, the bHLH transcription factors regulated triterpenoid metabolism by initiating $B p S S$ and $B P S E$ mRNA transcription by binding to the G-Box region [2]. In this study, the accumulation of squalene in INVScl1 transformants was analyzed by HPLC. After $12 \mathrm{~h}$ of cultivation, the contents of squalene in different INVScl1 transformants decreased in the following order: pYES-BpSS-BpbHLH9 $(0.328 \mathrm{mg} / \mathrm{g})>$ pYES3-BpbHLH9 $(0.165 \mathrm{mg} / \mathrm{g})>\mathrm{pYES} 2-\mathrm{BpSS}(0.134 \mathrm{mg} / \mathrm{g})>$ empty vector $(0.082 \mathrm{mg} / \mathrm{g} ;$ Fig. 8$)$. The total triterpenoid content in the INVScl-pYES-SS-bHLH9 yeast cells was $10.39 \%$ higher than that of the control yeast cells (INVScl-pYES2). The total triterpenoid contents of the INVScl-pYES2-SS and INVScl-pYES3-bHLH9 yeast cells were $4.49 \%$ and $6.00 \%$ higher, respectively, compared to the control (Fig. 9). The relative expression levels of $H M G R, F P S, S S, S E, B P Y$ and $B P W$ were up-regulated significantly in transgenic birch with overexpression BpbHLH9 (Fig. 10). In addition, the overexpression of BpbHLH9 enhanced the contents of betulinic acid, oleanolic acid and betulin in birch (Fig. 11). These results confirmed that BpbHLH9 was involved in the syntheses of squalene and triterpenoids. The main function of MYB transcription factor is to regulate secondary metabolism; it also plays a significant role in plant 
metabolism and development [32, 33]. In our study, the promoter sequences of BpMYC4 and BpbHLH9 were found to have an MBS cis-acting elements that served as an MYB-binding site. We hypothesize that BpMYC4 and $B p b H L H 9$ specifically bound MYB transcription factor to initiate the transcription of BpMYC4 and BpbHLH9 mRNA or the formation of bHLH/MYB complexes. Schaart et al. [34] reported the control of proanthocyanidin biosynthesis by MYB-bHLH-WD40 regulatory complexes in strawberry [34]. Zhao et al. [35] demonstrated that MYB-bHLH-WD4O proteins in Camellia sinensis controlled multiple enzymatic steps in the biosynthetic pathway of flavonoid production. Therefore, the results of this study suggested that MYB bound to BpbHLH9 and BpMYC4 in the MeJA signaling pathway to regulate triterpene synthesis in birch. Based on the results of this study and previous reports [2, 34, 35], we proposed a simplified working model for the role of BpbHLH9 in hormone-induced triterpenoid synthesis in B. platyphylla (Fig. 12). In this model, BpbHLH9 was induced by $\mathrm{ABA} / \mathrm{MeJA}$ in response to exogenous hormone signals but repressed by ABA/ethylene. BpbHLH9 interacted with MYB $[34,35]$ to initiate $B p S S$ expression by binding the G-box cis-acting elements of the $B p S S$ promoter [2], which subsequently regulated the synthesis of triterpenoids. The four hormones (ABA, ethylene, $\mathrm{ABA}$ and MeJA) mentioned above might have also directly regulated the expression of $B p S S$.

\section{Conclusions}

In this study, we cloned and isolated two genes, BpMYC4 and $B p b H L H 9$, that encoded transcription factors involved in triterpene synthesis. We analyzed the sequences of these genes using bioinformatics software and detected

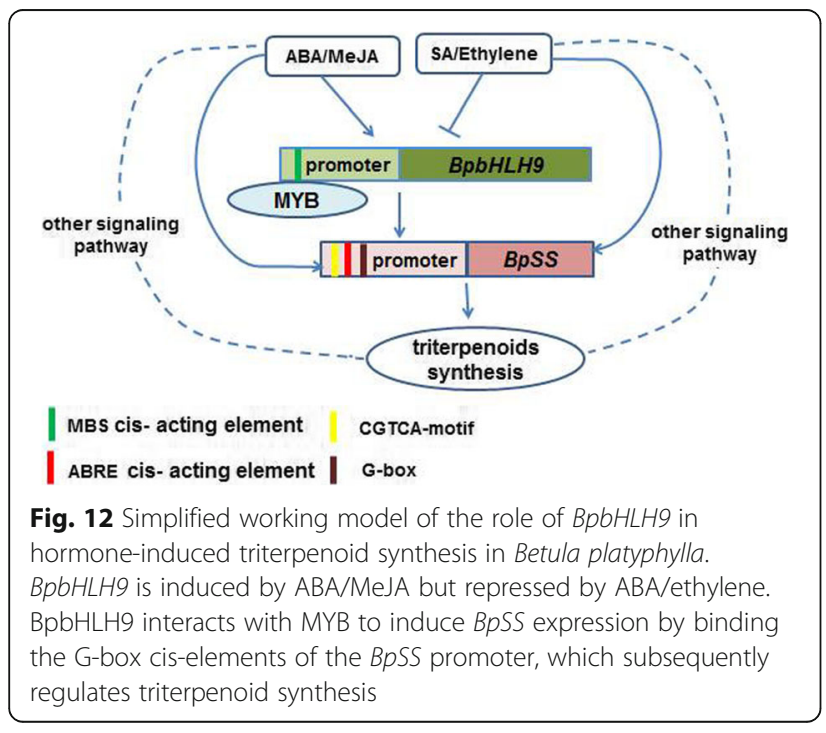

their expression patterns. BpbHLH9 increased the contents of squalene and total triterpenoids in yeast strains. The recombinant yeast strains lays the foundation for producing 2,3-oxidation squalene and its downstream products by synthetic biology method. Moreover, BpbHLH9 was involved in triterpenoid synthesis in birch, which could enhance the expression of key genes of triterpenoid pathway and the contents of oleanolic acid, betulinic acid and betulin. The findings provide useful information for future research on the bHLH regulation of terpene synthesis in transgenic birch.

\section{Methods}

\section{Plant and phytohormone treatment}

Different tissues from four-week-old birch seedlings were used in this study. For treatments with GA $(100 \mathrm{mg} / \mathrm{L})$, MeJA $(100 \mu \mathrm{M})$, SA $(50 \mathrm{mg} / \mathrm{L})$, ethephon $(5 \mathrm{mM})$, and ABA $(10 \mu \mathrm{M})$, the root of the plant was completely soaked in WPM liquid medium including the appropriate hormone solution. Birch sapling leaves and stems were harvested at different times after phytohormone treatment.

\section{Cloning and phylogenetic analysis of genes encoding MYC4 and bHLH9}

Complementary DNA and RNA were extracted from birch seedlings using the cetrimonium bromide method reported by Zhang et al. [2]. Using the birch cDNA treated with the above hormones as a template, a pair of degenerate oligonucleotide primers, MYC4-F/MYC4-R and bHLH9-F/bHLH9-R (Additional File 1: Table S1), were designed according to the transcriptome data of $B$. platyphylla recorded in our lab. Complete fragments of BpMYC4 and BpbHLH9 DNA were recovered, and the DNA was sequenced. Full-length nucleotide sequences of two genes were analyzed using bioinformatics software referring to the methods of Zhang et al. [2].

\section{Expression analysis of tissue-specific and phytohormone treatment of BpMYC4 and BpbHLH9}

The different tissues used to extract RNA were derived from hormone-treated birch. Total RNA was reversetranscribed into cDNA according to the user's manual (Takara, China). RT-PCR was carried out according to our previously reported method $[2,16]$. The raw data of relative quantification values were calculated according to Livak and Schmittgen with modification [36].

\section{Isolation of BpMYC4 and BpbHLH9 promoters}

Genomic DNA of B. platyphylla was extracted from fresh tissue. Genome walking products were constructed according to the manufacturer's protocols (Genome Walking $^{\text {Tu }}$ Kit, Takara). MYC4-SP1 with bHLH9-SP1, MYC4-SP2 with bHLH9-SP2, and MYC4-SP3 with bHLH9-SP3 (Additional File 1: Table S1) were used as 
the primers. The promoter sequences were cloned into the vector and subjected to nucleotide sequencing. The putative cis-acting elements of the promoters of $B p M Y C 4$ and BpbHLH9 were identified by consulting the PlantCARE database.

\section{Subcellular localization of BpMYC4 and BpbHLH9}

The full-length BpMYC4 and BpbHLH9 coding regions without termination codons were amplified using MYC4NcoI-F/R and bHLH9-NcoI-F/R (Additional File 1: Table S1) as primers, respectively. The coding sequences of the two genes were inserted in the Ncol sites of the pCAMBIA1303 vector (Biovector, USA) using an In-Fusion HD Cloning Kit (Takara, China). The fusion constructs pCAMBIA1303-BpMYC4 and pCAMBIA1303-BpbHLH9 and control pCAMBIA1303 were mobilized in strain LBA4404 using the triprental hybridization method. Agrobacterium-mediated transient expression in onion epidermal cells has been completed subcellular localization [37]. The infected inner skin of the onion was removed, and images were taken of the BpMYC4/GFP and BpbHLH9/GFP fusion proteins under a fluorescence microscope (Axioskop2plusFL, Zess, Germany).

\section{Identify of $B p b H L H 9$ gene in yeast INVScl1 strain}

The ORF of the BPbHLH9 PCR products was amplified using primers bHLH9-BamHI-F and bHLH9- BamHI-R (Additional File 1: Table S1) and inserted into the corresponding BamHI sites of pYES3 (Invitrogen) to construct the fusion plasmid pYES3-BpbHLH9. The plasmid was transferred to the INVScl1 strain (Clontech, USA) and special INVScl1 strains bearing pYES2-BpSS, which were created in our laboratory using the PEG/LiAc method. Single colonies on the corresponding defective medium (SC- Trp and SC-Ura-Trp)were randomly selected and transferred to the corresponding liquid medium to expand the culture. The cells were collected, and the yeast DNA was extracted and BpbHLH9 gene detected by PCR.

\section{Squalene accumulation in INVScl1 transformants}

The transformants were cultured, and the expression of BpbHLH9 was induced as described in Zhang et al. [2]. The cellular squalene was extracted as reported in Lu et al. [38], and the squalene content was analyzed by HPLC (Waters 1525-2489) [2]. The test was repeated three times.

\section{Analysis of total triterpenoid content in INVScl1 transformants}

The transformants of BpbHLH9 were induced as described in Zhang et al. [2], and the cells were then collected and dried. Dried yeast cells $(50 \mathrm{mg})$ were weighed and placed in $4 \mathrm{~mL}$ of $95 \%$ ethanol solution for $24 \mathrm{~h}$. The specific extraction and detection methods for total triterpenoids are found in Ref. [16].
Determination of betulinic acid, oleanolic acid and betulin in transgenic birch

Dried transgenic birch callus was ground into a powder, and $0.50 \mathrm{~g}$ of powder was soaked in $20 \mathrm{~mL}$ hydrochloric acid:ethanol solution (2:8 by volume). The solution was refluxed for $3 \mathrm{~h}$ in a water bath at $90{ }^{\circ} \mathrm{C}$. After filtration, $15 \mathrm{~mL}$ of distilled water was added to $15 \mathrm{~mL}$ of the filtrate, and the ethanol was completely distilled off in a water bath at $80{ }^{\circ} \mathrm{C}$. The mixture was extracted three times with $20 \mathrm{~mL}$ of ether. The combined extracts were evaporated to dryness at $40{ }^{\circ} \mathrm{C}$ and dissolved in $2.5 \mathrm{~mL}$ methanol for HPLC analysis.

\section{Additional files}

Additional file 1: Table S1. List of primers used in the study. (DOCX $16 \mathrm{~kb}$ ) Additional file 2: Figure S1. Nucleotide sequence and deduced amino acid sequence of BPMYC4 from Betula platyphylla. (DOCX $287 \mathrm{~kb}$ )

Additional file 3: Figure S2. Nucleotide sequence and deduced amino acid sequence of BpbHLH9 from Betula platyphylla. (DOCX 193 kb)

Additional file 4: Figures S3 and S4. Promoter sequences of the BPMYC4 and BpbHLH9 from birch. (DOCX $137 \mathrm{~kb}$ )

Additional file 5: Figures S5, S6 and S7. Identification and acquisition of BpbHLH9 transgenic seedlings. (DOCX $730 \mathrm{~kb}$ )

\section{Abbreviations}

ABA: Abscisic acid; bHLH: Basic helix-loop-helix; FPS: Farnesyl pyrophosphonate synthase; GA: Gibberellin; HMGR: 3-hydroxy-3-methyl glutaryl coenzyme A reductase; HPLC: High-performance liquid chromatography; MeJA: Methyl jasmonate; SA: Salicylic acid; SE: Squalene epoxidase; SS: Squalene synthase

\section{Acknowledgments}

The authors greatly acknowledge professor You Xiangling at Northeast Forestry University for providing seeds of birch. The authors also thank professor Hu Shanglianof at Southwest University of Science and Technology for providing the victor.

\section{Funding}

This work was supported by the National Natural Science Foundation of China (31570589), the Fundamental Research Fund for the Central Universities (257-2017DY02), and the National Basic Science Training Fund (J1210053). The funding bodies of the research is Chinese government and Ministry of Education of China, and they providing funds play very important roles in experiment test and data collection, data analysis of study, and in native English writing of manuscript and publish fee.

Availability of data and materials

All the supporting data are included as additional files.

Authors' contributions

$J Y$ and YGZ designed and guided this study, participated in the gene cloning and genetic transformation of birch and modified the manuscript; $X \mathrm{~L}$ performed amount of the experiment, including Cloning and expression of BpMYC4 and BpbHLH9 genes, Obtaining transgenic birch and identification and wrote the manuscript; YL completed subcellular localization of BpMYC4 and BpbHLH9 and data collection, and ZYQ participated in gene promoter cloning and sequence analysis; LS contributed to gene expression analysis and data collection, and SYW completed role of BpbHLH9 in triterpenoid synthesis in yeast, data collection and analysis; JY and JLX performed squalence product and triterpenoids content analysis using HPLC and data collection and analysis. All authors have read and approved the manuscript. 


\section{Ethics approval and consent to participate}

The source of the plant materials (birch seedlings) used in our study come from our lab(Forest Biological Engineering Laboratory, College of Life Science, Northeast Forestry University, Harbin, China; Professor Yaguang Zhan is the chairwomen of the laboratory, Email: yaguangzhan@126.com). All plants materials are allowed to be used and provided free of charge in this study. The birch seedlings used in this experiment come from tissue culture seedlings in our laboratory, its not endangered materials or species.

\section{Consent for publication}

Not applicable.

\section{Competing interests}

The authors declare that they have no competing interests.

\section{Publisher's Note}

Springer Nature remains neutral with regard to jurisdictional claims in published maps and institutional affiliations.

\section{Author details}

${ }^{1}$ College of Life Science, Northeast Forestry University, Harbin 150040, China. ${ }^{2}$ State Key Laboratory of Tree Genetic Breeding, Northeast Forestry University, Harbin 150040, China. ${ }^{3}$ College of Agriculture, Northeast Agriculture University, Harbin 150010, China.

\section{Received: 27 March 2017 Accepted: 2 November 2017}

\section{Published online: 21 November 2017}

\section{References}

1. Yin J, Li C, Zhan Y, Sun H, Gong Y, Zhang M, Xiao J. The response of physiological characteristics, expression of OSC genes, and accumulation of triterpenoids in Betula platyphylla Sukto MeJA and SA treatment. Plant Mol Biol Rep. 2015;34(2):427-39.

2. Zhang M, Wang S, Yin J, Li C, Zhan Y, Xiao J, Liang T, Li X. Molecular cloning and promoter analysis of squalene synthase and squalene epoxidase genes from Betula platyphylla. Protoplasma. 2016;5:1347-63.

3. Huang D, Dai W. Molecular characterization of the basic helix-loop-helix $(b H L H)$ genes that are differentially expressed and induced by iron deficiency in Populus. Plant Cell Rep. 2015;34(7):1211-24.

4. Mertens J, Pollier J, Vanden BR, López-Vidriero I, Franco-Zorrilla JM, Goossens A. The bHLH transcription factors TSAR1 and TSAR2 regulate triterpene saponin biosynthesis in Medicago truncatula. Plant Physiol. 2016; 170(1):194-210.

5. Yang CQ, Fang X, XM W, Mao YB, Wang $L$, Chen XY. Transcriptional regulation of plant secondary metabolism. J Integ. Plant Biol. 2012;54(10):703-12.

6. Kazan K, Manners JM. MYC2: the master in action. Mol Plant. 2013;6(3):686-703.

7. Zhang $H$, Hedhili S, Montiel G, Zhang Y, Chatel G, Pré M, Gantet P, Memelink J. The basic helix-loop-helix transcription factor CrMYC2 controls the jasmonate-responsive expression of the ORCA genes that regulate alkaloid biosynthesis in Catharanthus roseus. Plant J. 2011;67(1):61-71.

8. Hong GJ, Xue XY, Mao YB, Wang LJ, Chen XY. Arabidopsis MYC2 interacts with DELLA proteins in regulating sesquiterpene synthase gene expression. Plant Cell. 2012;24(6):2635-48.

9. Ji Y, Xiao J, Shen Y, Ma D, Li Z, Pu G, Li X, Huang L, Liu B, Ye H. Cloning and characterization of AabHLH1, a bHLH transcription factor that positively regulates artemisinin biosynthesis in Artemisia annua. Plant Cell Physiol. 2014:55(9):1592-604

10. Spyropoulou EA, Haring MA, Schuurink RCRNA. Sequencing on Solanum lycopersicum trichomes identifies transcription factors that activate terpene synthase promoters. BMC Genomics. 2014;15(1):402.

11. Gonzalez A, Zhao M, Leavitt JM, Lloyd AM. Regulation of the anthocyanin biosynthetic pathway by the TTG1/bHLH/Myb transcriptional complex in Arabidopsis seedlings. Plant J. 2008;53(5):814-27.

12. Shoji T, Hashimoto T. Tobacco MYC2 regulates jasmonate-inducible nicotine biosynthesis genes directly and by way of the NIC2-locus ERF genes. Plant Cell Physiol. 2011;52(6):1117-30

13. Shang $Y$, Ma $Y$, Zhou Y, Zhang H, Duan L, Chen H, Zeng J, Zhou Q, Wang S, Gu W. Biosynthesis, regulation, and domestication of bitterness in cucumber. Science. 2014;346(6213):1084-8.

14. Moerkercke AV, Steensma P, Schweizer F, Pollier J, Gariboldi I, Payne R, Bossche RV, Miettinen K, Espoz J, Purnama PC. The bHLH transcription factor BIS1 controls the iridoid branch of the monoterpenoid indole alkaloid pathway in Catharanthus roseus. Proc Natl Acad Sci USA. 2015;112(26):8130-5.

15. Todd AT, Liu E, Polvi SL, Pammett RT, Page JE. A functional genomics screen identifies diverse transcription factors that regulate alkaloid biosynthesis in Nicotiana Benthamiana. Plant J. 2010;62(4):589-600.

16. Yin J, Ren CL, Zhan YG, Li CX, Xiao JL, Qiu W, Li XY, Peng HM. Distribution and expression characteristics of triterpenoids and OSC genes in white birch (Betula platyphylla suk.). Mol Biol Rep. 2013;39(3):2321-8.

17. Yin JJ, Liang T, Wang S, Zhang M, Xiao J, Zhan Y, Li C. Effect of drought and nitrogen on betulin and oleanolic acid accumulation and OSC gene expression in white birch saplings. Plant Mol Biol Rep. 2014;33(3):705-15.

18. Zhou M, Memelink J. Jasmonate-responsive transcription factors regulating plant secondary metabolism. Biotechnol Adv. 2016;34(4):441-9.

19. Moerkercke AV, Steensma P, Gariboldi I, Espoz J, Purnama PC, Schweizer F Miettinen K, Bossche RV, Clerca R, Memelink J. The basic helix-loop-helix transcription factor BIS2 is essential for monoterpenoid indo aloid production in the medicinal plant Catharanthus roseus. Plant J. 2016;88(1):3.

20. Sablowski RW, Moyano E, Culianez-Macia FA, Schuch W, Martin C, Bevan MA. Flower-specific Myb protein activates transcription of phenylpropanoid biosynthetic genes. EMBO J. 1994;13(1):128-37.

21. Nie $X$, Zhao $Y$, Zhang L, Tang $Y$, Wang J, Zhao Z. Mechanisms of MYB-bHLHWD40 complex in the regulation of Anthocyanin biosynthesis in plants. Agric Biotechnol. 2015;4(3):5-8

22. Abe H, Urao T, Ito T, Seki M, Shinozaki K. Arabidopsis AtMYC2 (bHLH) and AtMYB2 (MYB) function as transcriptional activators in abscisic acid signaling. Plant Cell. 2003;15(1):63-78.

23. Creelman RA, Mullet JE. Jasmonic acid distribution and action in plants: regulation during development and response to biotic and abiotic stress. Proc Natl Acad Sci USA. 1995;92:4114-9.

24. Nazarli H, Ahmadi A, Hadian J. Salicylic acid and methyl jasmonate enhance drought tolerance in chamomile plants. J Herb Med Pharmacol. 2014;3(2): 87-92.

25. Gundlach H, Muller MJ, Kutchan TM, Zenk MH. Jasmonic acid is a signal transducer in elicitorinduced plant cell cultures. Proc Natl Acad Sci U S A. 1992;89(6):2389-93.

26. Chatel G, Montiel G, Pré M, Memelink J, Thiersault M, Saintpierre B, Doireau P, Gantet P. CrMYC1, a Catharanthus roseus elicitor-and jasmonateresponsive bHLH transcription factor that binds the G-box element of the strictosidine synthase gene promoter. J Exp Bot. 2003;54(392):2587-8.

27. Memelink J, Gantet P. Transcription factors involved in terpenoid indole alkaloid biosynthesis in Catharanthus roseus. Phytochem Rev. 2007;6(2):353-62.

28. Li R, Weldegergis BT, Li J, Jung C, Qu J, Sun Y, Qian H, Tee C, van Loon JJ, Dicke M. Virulence factors of geminivirus interact with MYC2 to subvert plant resistance and promote vector performance. Plant Cell. 2014;26(12):4991-5008.

29. Vand DD, Leon-Reyes A, Koornneef A, Van Verk MC, Rodenburg N, Pauwels L, Goossens A, Körbes AP, Memelink J, Ritsema T. Salicylic acid suppresses jasmonic acid signaling downstream of SCF ${ }^{\mathrm{CO} 11}$-JAZ by targeting GCC promoter motifs via transcription factor ORA59. Plant Cell. 2013;25(2):744-61.

30. El-Kereamy A, Chervin C, Roustan JP, Cheynier V, Souquet JM, Moutounet M, Raynal J, Ford C, Latché A, Pech JC. Exogenous ethylene stimulates the long term expression of genes related to anthocyanin biosynthesis in grape berries. Physiol Plantarum. 2003;119(2):175-282.

31. Pré M, Siberil Y, Memelink J, Champion A, Doireau P, Gantet P. Isolation by the yeast onehybrid system of cDNAs encoding transcription factors that bind to the G-box element of the strictosidine synthase gene promoter from Catharanthus roseus. Int J Biochromatogr. 2000;5(3):229-44.

32. Dubos C, Le GJ, Baudry A, Huep G, Lanet E, Debeaujon I, Routaboul JM, Alboresi A, Weisshaar B, Lepiniec L. MYBL2 is a new regulator of flavonoid biosynthesis in Arabidopsis thaliana. Plant J. 2008;55(6):940-53.

33. Shen $H$, He XZ, Poovaiah CR, Wuddineh WA, Ma JY, Mann DGJ, Wang HZ, Jackson L, Tang YH, Stewart CN. Functional characterization of the switchgrass (Panicum virgatum) R2R3-MYB transcription factor PvMYB4 for improvement of lignocellulosic feedstocks. New Phytol. 2012;193(1):121-36.

34. Schaart JG, Dubos C, Irene RDLF, Houwelingen AMML, Vos RCH, Jonker HH, Xu W, Routaboul JM, Lepiniec L, Bovy AG. Identification and characterization of MYB-bHLH-WD40 regulatory complexes controlling proanthocyanidin biosynthesis in strawberry (Fragariax ananassa) fruits. New Phytol. 2013; 197(2):454-67.

35. Zhao L, Gao L, Wang $H$, Chen X, Wang Y, Yang $H$, Wei C, Wan X, Xia T. The R2R3-MYB, bHLH, WD40, and related transcription factors in flavonoid biosynthesis. Funct Integr Genomic. 2013;13(1):75-98. 
36. Livak K, Schmittgen TD. Analysis of relative gene expression data using real-time quantitative PCR and the $2^{-\triangle \Delta C T}$ method. Methods. 2001;25(4): 402-8.

37. Nagegowda DA. Plant volatile terpenoid metabolism: biosynthetic genes, transcriptional regulation and subcellular compartmentation. FEBS Lett. 2010;584(14):2965-73.

38. HT L, Jiang Y, Chen F. Determination of squalene using high performance liquid chromatography with diode array detection. Chromatographia. 2004; 59(5):367-71.

Submit your next manuscript to BioMed Central and we will help you at every step:

- We accept pre-submission inquiries

- Our selector tool helps you to find the most relevant journal

- We provide round the clock customer support

- Convenient online submission

- Thorough peer review

- Inclusion in PubMed and all major indexing services

- Maximum visibility for your research

Submit your manuscript at www.biomedcentral.com/submit 